\title{
Beneficial effects of anisodamine in shock involved cholinergic anti-inflammatory pathway
}

\author{
Ting Zhao, Dong-Jie Li, Chong Liu, Ding-Feng Su and Fu-Ming Shen* \\ Department of Pharmacology, School of Pharmacy, Second Military Medical University, Shanghai, China
}

\section{Edited by:}

Xue-Jun Sun, Taishan Medical College,

China

\section{Reviewed by:}

Kayo Masuko, Sagami Women's

University, Japan

Keliang Xie, General Hospital of Tianjin

Medical University, China

*Correspondence:

Fu-Ming Shen, Department of

Pharmacology, School of Pharmacy,

Second Military Medical University, 101

Guo He Road, Shanghai 200433,

China.

e-mail: fumingshen@hotmail.com
Anisodamine, an antagonist of muscarinic receptor, has been used therapeutically to improve blood flow in circulatory disorders such as septic shock in China since 1965. The main mechanism of anisodamine for anti-shock proposed in Pharmacology for Chinese medical students is to improve blood flow in the microcirculation. Here, we suggest a new mechanism for its anti-shock effect. That is, anisodamine, by blocking muscarinic receptor, results in rerouting of acetylcholine to $\alpha 7$ nicotinic acetylcholine receptor ( $\alpha 7 \mathrm{nAChR}$ ) bringing about increased acetylcholine-mediated activation of $\alpha 7 \mathrm{nAChR}$ and the cholinergic anti-inflammatory pathway.

Keywords: anisodamine, acetylcholine, $\alpha 7 \mathrm{nAChR}$, inflammation, shock
Anisodamine, a belladonna alkaloid isolated from the Chinese medicinal herb Scopolia tangutica Maxim (Figure 1) of the Solanaceae family indigenous to Tibet and first synthesized by Chinese scientists in 1975, is an ester formed by the combination of tropic acid and the organic base tropine, and is related to atropine with a hydroxyl group at the asymmetric carbon position six of the tropine radical (Figure 2). It is generally considered as an antagonist of muscarinic receptor in the textbook of Pharmacology for medical students in China (Yang and Su, 2008), and has been used therapeutically to improve blood flow in circulatory disorders such as septic shock and disseminated intravascular coagulation since 1965. Studies found that anisodamine significantly reduced the mortality rate of toxic bacillary dysentery from $20-30$ to $0.5 \%$ and fulminant epidemic meningitis from 66.9 to $12.4 \%$ in humans (Anonymous, 1975; Wang and Kuo, 1978; Xiu, 1980). In different kinds of shock models prepared from either rabbit, or cat, or rat, induced by late hemorrhage, superior mesenteric artery occlusion, septic shock from peritonitis, and traumatic shock, anisodamine significantly alleviated the progress of shock and increased the survival rate of the animals than that of other commonly used vasoactive drugs, such as norepinephrine, phenoxybenzamine, dopamine, and aramine (Hock et al., 1983; Su et al., 1983, 1984).

Various mechanisms for the anti-shock action of anisodamine were proposed. As a vasodilator, it was believed to be a valuable adjunct to the resuscitation regime of burn shock, and a promising drug to abate endogenous endotoxemia subsequent to splanchnic vasoconstriction due to hypovolemia in burn patients (Sheng et al., 1997). It was also demonstrated that the efficacy of anisodamine in bacteremic shock was mediated by inhibition of granulocyte, and platelet aggregation and thromboxane synthesis (Xiu et al., 1982), and anisodamine protected tissues from reperfusion damage by inhibition of thromboxane synthesis after resuscitation in burn shock patients (Huang et al., 1990). Other possible mechanisms included fibrinolytic effects, reversal of endotoxin induced vascular leakage, stabilization of lysosomes and cathepsin inhibition (Poupko et al., 2007). These actions of anisodamine resulted in the ultimate improvement of blood flow in the microcirculation, which is the main mechanism proposed in the textbook of Pharmacology.

Ruan et al. (2001) found that anisodamine was able to counteract endothelial cell activation by inhibiting lipopolysaccharide-induced plasminogen activator inhibitor- 1 and tissue factor expression possibly through the NF- $\kappa \mathrm{B}$ pathway, and suggested that the ability of anisodamine to counteract lipopolysaccharide effects on endothelial cells might be one underlying mechanism for its efficacy in the treatment of bacteremic shock. It is interesting because NF- $\kappa \mathrm{B}$ is a key role in inflammatory diseases, which is able to regulate the synthesis of pro-inflammatory cytokines, such as tumor necrosis factor-alpha (TNF- $\alpha$ ), interleukin-1 beta (IL-1 $\beta$ ), IL-6, and IL-8 (Baldwin, 1996; Tak and Firestein, 2001). These suggested that the anti-shock effect of anisodamine might be related to anti-inflammation.

The cholinergic anti-inflammatory pathway was a recently proposed pathway, which links the vagus nerve and immune system through $\alpha 7$ nicotinic acetylcholine receptor $(\alpha 7 \mathrm{nAChR})$. That is, the vagus nerve can modulate the immune response and inhibit inflammation through a physiological mechanism, which can be translated into a pharmacological strategy. Acetylcholine, the principal neurotransmitter of the vagus nerve, signaling through $\alpha 7 \mathrm{nAChR}$, can inhibit the production of pro-inflammatory cytokines from macrophages (Libert, 2003; Wang et al., 2003; Figure 3). Our previous study evaluated the effect of anisodamine, the muscarinic receptor antagonist, in rodent models of endotoxic shock. We hypothesized that blocking muscarinic receptor with anisodamine resulted in rerouting of acetylcholine to $\alpha 7 \mathrm{nAChR}$ bringing about increased acetylcholine-mediated activation of $\alpha 7 \mathrm{nAChR}$ and the cholinergic anti-inflammatory pathway. It was found that methyllycaconitine (an selective $\alpha 7 \mathrm{nAChR}$ antagonist) significantly antagonized the beneficial effect of anisodamine on 


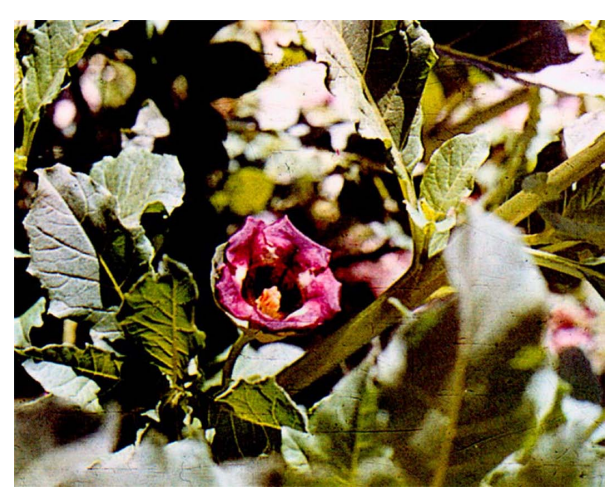

FIGURE 1 |The Chinese medicinal herb Scopolia tangutica Maxim.

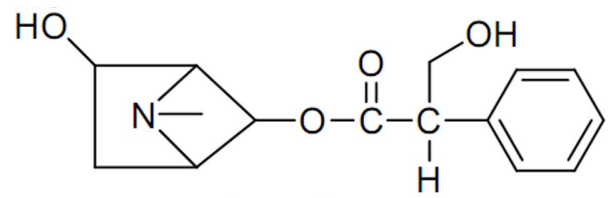

Anisodamine

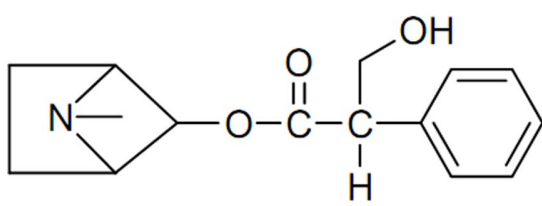

Atropine

FIGURE 2 | Chemical structures of anisodamine and atropine.

mean arterial pressure and TNF- $\alpha$, interleukin- $1 \beta$ expression in response to lipopolysaccharide; the anti-shock effects of anisodamine were markedly attenuated in vagotomized mice and $\alpha 7 \mathrm{nAChR}^{-1-}$ mice; in vitro, anisodamine significantly augmented the effect of acetylcholine on fluorescence intensity stained with fluorescein isothiocyanate-labeled- $\alpha$-bungarotoxin and TNF- $\alpha$ production stimulated with lipopolysaccharide. Our results suggest that activation of the vagal immune reflex can not only be

\section{REFERENCES}

Anonymous. (1975). Anisodamine in treatment of some diseases with manifestations of acute microcirculatory insufficiency. Chin. Med. J. 1, 127-132.

Baldwin, A. S. Jr. (1996). The NF-kappa B and I kappa B proteins: new discoveries and insights. Annu. Rev. Immunol. 14, 649-683.

Hock, C. E., Su, J. Y., and Lefer, A. M. (1983). Salutary effects of anisodamine in murine traumatic shock. Circ. Shock 11, 211-216.

Huang, Y.S., Li, A., and Yang, Z. C. (1990). Roles of thromboxane and its inhibitor anisodamine in burn shock. Burns $16,249-253$.
Li, D. J., Evans, R. G., Yang, Z. W., Song, S. W., Wang, P., Ma, X. J., Liu, C., Xi, T., Su, D. F., and Shen, F. M. (2011). Dysfunction of the cholinergic anti-inflammatory pathway mediates organ damage in hypertension. Hypertension 57, 298-307.

Libert, C. (2003). Inflammation: a nervous connection. Nature 421, 328-329.

Liu, C., Shen, F. M., Le, Y. Y., Kong, Y., Liu, X., Cai, G. J., Chen, A. F., and Su, D. F. (2009). Anti-shock effect of anisodamine involves a novel pathway for activating a7 nicotinic acetylcholine receptor. Crit. Care Med. 37, 634-641.

Poupko, J. M., Baskin, S. I., and Moore, E. (2007). The pharmacological

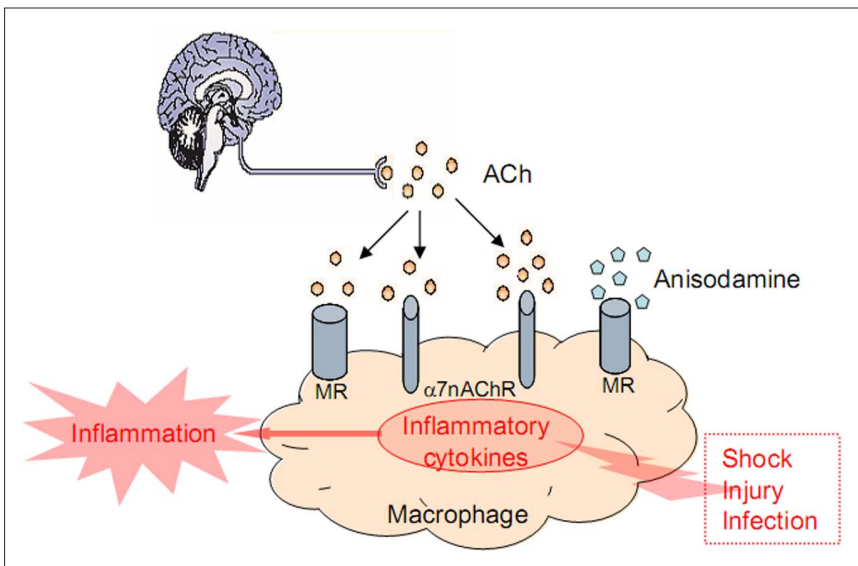

FIGURE 3 |Action mode of anisodamine in cholinergic anti-inflammatory pathway.

pursued by stimulation of the vagus nerve or $\alpha 7 \mathrm{nAChR}$ but also by inhibition of muscarinic receptors, which results in secondary activation of the cholinergic anti-inflammatory pathway (Liu et al., 2009; van Westerloo, 2009; Figure 3). We also found that $\alpha 7 \mathrm{nAChR}$ played an important role in the organ damage in spontaneously hypertensive rats (SHRs), and chronic treatment of SHRs with the $\alpha 7 \mathrm{nAChR}$ agonist PNU-282987 relieved end organ damage and inhibited tissue levels of pro-inflammatory cytokines involved the NF- $\kappa$ B pathway (Li et al., 2011). These suggest that the cholinergic anti-inflammatory pathway might act, at least partly, through the NF- $\kappa \mathrm{B}$ pathway, and anisodamine can indirectly activate the cholinergic anti-inflammatory pathway by blocking muscarinic receptor.

In summary, we suggest that the beneficial effect of anisodamine in shock involves the cholinergic anti-inflammatory pathway, which expands the possibilities of using the cholinergic anti-inflammatory pathway for clinical benefit. However, further studied are needed on the molecular and preclinical level.

\section{ACKNOWLEDGMENTS}

This work was supported by grants from the National Natural Science Foundation of China (30870940, 81070118), the Science and Technology Development Foundation of Shanghai (08JC1406000), and the Shanghai Municipal Education Commission (10ZZ52).

properties of anisodamine. J. Appl. Toxicol. 27, 116-121.

Ruan, Q. R., Zhang, W. J., Hufnagl, P., Kaun, C., Binder, B. R., and Wojta, J. (2001). Anisodamine counteracts lipopolysaccharide-induced tissue factor and plasminogen activator inhibitor-1 expression in human endothelial cells: contribution of the NF-kB pathway. J. Vasc. Res. 38, 13-19.

Sheng, C. Y., Gao, W. Y., Guo, Z. R., and He, L. X. (1997). Anisodamine restores bowel circulation in burn shock. Burns 23, 142-146.

Su, J. Y., Hock, C. E., and Lefer, A. M (1984). Beneficial effect of anisodamine in hemorrhagic shock. Naunyn
Schmiedebergs Arch. Pharmacol. 325, 360-365.

Su, J. Y., Wu, L., and Tang, C. (1983). Experimental study in rabbits of the antishock effect of anisodamine (654-2), and its mechanism of action. Resuscitation 10, 173-184.

Tak, P. P., and Firestein, G. S. (2001). NF-kB: a key role in inflammatory diseases. J. Clin. Invest. 107, 7-11.

van Westerloo, D. J. (2009). A new approach to step on the vagal antiinflammatory gas pedal. Crit. Care Med. 37, 778-779.

Wang, H., Yu, M., Ochani, M., Amella, C. A., Tanovic, M., Susarla, S., Li, J. H., Yang, H., Ulloa, L., Al-Abed, Y., Czura, 
C. J., and Tracey, K. J. (2003). Nicotinic acetylcholine receptor alpha7 subunit is an essential regulator of inflammation. Nature 421, 384-388.

Wang, S. T., and Kuo, N. L. (1978). Experience in emergency treatment of shock due to infection. Chin. Med. J. 6, 497-500.

Xiu, R. J. (1980). Studies on microcirculation in the Institute of Basic Medical Sciences, Chinese Academy Medical Science. Microvasc. Res. 20, 371-373.
Xiu, R. J., Hammerschmidt, D. E., Coppo, P. A., and Jacob, H.S. (1982). Anisodamine inhibitsthromboxane synthesis, granulocyte aggregation, and platelet aggregation: a possible mechanism for its efficacy in bacteremic shock. JAMA247,1458-1460. Yang,B.F., and Su,D.F.(2008). "Antagonists of muscarinic receptors," in Pharmacology, 7th Edn, C. Y. Zhang (Beijing: People's Medical Publishing House), 72-79.

Conflict of Interest Statement: The authors declare that the research was conducted in the absence of any commercial or financial relationships that could be construed as a potential conflict of interest.

Received: 31 January 2011; paper pending published: 09 February 2011; accepted: 11 April 2011; published online: 02 May 2011.

Citation: Zhao T, Li D-J, Liu C, Su $D-F$ and Shen F-M (2011) Beneficial effects of anisodamine in shock involved cholinergic anti-inflammatory pathway.
Front. Pharmacol. 2:23. doi: 10.3389/ fphar.2011.00023

This article was submitted to Frontiers in Ethnopharmacology, a specialty of Frontiers in Pharmacology.

Copyright (C) 2011 Zhao, Li, Liu, Su and Shen. This is an open-access article subject to a non-exclusive license between the authors and Frontiers Media SA, which permits use, distribution and reproduction in other forums, provided the original authors and source are credited and other Frontiers conditions are complied with. 